\title{
Non-deterministic computation and the Jayne-Rogers Theorem
}

\author{
Arno Pauly \\ Computer Laboratory \\ University of Cambridge, United Kingdom \\ Arno.Pauly@cl.cam.ac.uk
}

\author{
Matthew de Brecht \\ National Institute of Information and Communications Technology \\ Kyoto, Japan \\ matthew@nict.go.jp
}

\begin{abstract}
We provide a simple proof of a computable analogue to the Jayne-Rogers Theorem from descriptive set theory. The difficulty of the proof is delegated to a simulation result pertaining to non-deterministic type- 2 machines. Thus, we demonstrate that developments in computational models can have applications in fields thought to be far removed from it.
\end{abstract}

\section{Introduction}

Non-deterministic type-2 machines (NDTMs) were suggested by Ziegler [34, 35, 33] as a model for hypercomputation in computable analysis. As demonstrated by Brattka, de Brecht and Pauly [2, 6], the strength of various kinds of type-2 non-deter-minism neatly classifies various important classes of noncomputable functions; and a characterization of such classes as those functions computable by certain NDTMs opens up new, simple ways to prove closure properties for them.

A NDTM with advice space $Z$ is a Turing machine with an input tape, an oracle tape, some work tapes and a write-once output tape. The input is an infinite sequence written on the input tape, the oracle tape is initialized with a guess, an infinite sequence from the set $Z$. The machine either halts eventually, which is seen as a rejection of the guess, or continues to write an infinite sequence on the output tape. For any valid input there must be an acceptable guess.

Thus, a NDTM naturally computes a multivalued function $f: \subseteq\{0,1\}^{\mathbb{N}} \rightrightarrows\{0,1\}^{\mathbb{N}}$. The notion of non-deter-ministic computability is then lifted to arbitrary represented spaces: Some $f: \mathbf{X} \rightrightarrows \mathbf{Y}$ is nondeterministally computable with advice space $Z$, iff there is an NDTM such that any $p \in\{0,1\}^{\mathbb{N}}$ denoting an element of $\mathbf{X}$ is accepted, and every successful computation produces a name for some $y \in f(x)$.

The power of NDTMs severely depends on the advice space. The spaces $\{0,1\}^{\mathbb{N}}$ and $\mathbb{N}$ yield incomparable computational power, $\mathbb{N} \times\{0,1\}^{\mathbb{N}}$ is more powerful than both, and $\mathbb{N}^{\mathbb{N}}$ again significantly more powerful than $\mathbb{N} \times\{0,1\}^{\mathbb{N}}$. The crucial property for us is that the additional computational power of $\mathbb{N} \times\{0,1\}^{\mathbb{N}}$ over $\mathbb{N}$ only applies to multivalued functions-any single-valued $f: \mathbf{X} \rightarrow \mathbf{Y}$ (with $\mathbf{Y}$ computably admissible) non-deter-ministically computable with advice space $\mathbb{N} \times\{0,1\}^{\mathbb{N}}$ already is non-deter-ministically computable with advice space $\mathbb{N}$.

We will apply the theory of non-deter-ministic computations to descriptive set theory. A subset of a metric space is called $\Delta_{2}^{0}$, if it is both the countable union of closed sets and the countable intersection of open sets. A function is called $\Delta_{2}^{0}$-measurable, iff the preimage of any open set is a $\Delta_{2}^{0}$-set. A function will be called $\mathscr{A}$-piecewise continuous, iff there is a countable cover of its domain by closed sets, such that the restriction to any such closed set is continuous.

Theorem 1 (Jayne \& Rogers [14]). Let $\mathbf{X}, \mathbf{Y}$ be metric spaces. If $\mathbf{X}$ is absolute Souslin- $\mathfrak{F}$, then $f: \mathbf{X} \rightarrow \mathbf{Y}$ is $\Delta_{2}^{0}$-measurable if and only if it is $\mathscr{A}$-piecewise continuous.

B. Löwe, G. Winskel (Eds.):

Developments in Computational Models 2012 (DCM 2012)

EPTCS 143, 2014, pp. 87-96, doi 10.4204/EPTCS.143.8 (c) A. Pauly and M. de Brecht

This work is licensed under the Creative Commons Attribution License. 
For a definition of absolute Souslin- $\mathfrak{F}$, see [16, Definition 25.4]. This criterion is not needed in the computable case, hence we do not give details here.

After the original proof by Jayne and Rogers [14], simplifications were provided first by Solecki [27] and then by Motto Ros and Semmes [18 $]^{1}$. While showing that piecewise continuous functions are $\Delta_{2}^{0}$ measurable is straight-forward, the known proofs for the other direction are all somewhat complicated, and non-constructive: The assumption that a function was both $\Delta_{2}^{0}$-measurable and not piecewise continuous is taken to a contradiction; there is no construction given for the countable closed cover witnessing the piecewise continuity of a $\Delta_{2}^{0}$-measurable function.

Working in the framework of computable analysis, we will provide a computable version of Theorem 1. that is we show how to compute information identifying a function as piecewise continuous from information identifying it as $\Delta_{2}^{0}$-measurable. This should not be confused with giving an analogue to Theorem 1 in effective descriptive set theory, which is investigated in Section 6 .

\section{Non-deterministic type-2 machines}

It is well-established (Weihrauch [32]) that a good model for computation on objects from analysis is given by Type- 2 machines. These essentially are the usual (deterministic) Turing machines with new semantics: The computation runs forever, hence every cell of the infinite tapes is actually accessible. A designated output tape allows the head only to move to the right, so any symbol to the left of the current head position remains unchanged. A well-behaved computation will write on the output infinitely often, and thus produces an infinite output sequence. Type- 2 machines should not be confused with the Infinite Time Turing Machines proposed by Hamkins and Lewis [10]—both the definitions and their purpose differ significantly.

Just as Type-2 machines are derived from deterministic Turing machines, we can derive non-deterministic Type-2 machines from non-deter-ministic Turing machines. We assume that the non-deter-minism is localized, i.e., that all non-deter-ministic bits are guessed at once. As a Type-2 machine has unlimited time available for the verification, it can utilize an infinite sequence of non-deter-ministic bits. In addition, we provide our machines with the promise that the guess-sequence is in some fixed set $Z \subseteq\{0,1\}^{\mathbb{N}}$-a property not relevant in the classical case.

A crucial difference between Type- 1 and Type-2 non-deter-minism is that the latter increases the computational power, whereas non-deter-ministic Turing machines can be simulated by deterministic ones. A typical example of a non-computable problem solvable by a NDTM (with advice space $Z=$ $\{0,1\}^{\mathbb{N}}$ ) is Weak König's Lemma, i.e., the problem to find an infinite path through an infinite binary tree. The binary tree could be given via its characteristic function, so for any vertex we can decide whether or not its left and/or right child are present in the tree, too.

A NDTM solving Weak König's Lemma guesses a potential path through the tree (as an infinite binary sequence), and proceeds to output the guessed sequence, while simultaneously checking that every vertex used is actually present in the tree. If the path is invalid, it uses some non-existent vertex, which will be detected and result in the rejection of the guess. As every infinite binary tree has an infinite path, there is some valid path which will be given as output without rejection.

As long as the advice space is fixed to $Z=\{0,1\}^{\mathbb{N}}$, any non-computable problem solvable by a corresponding NDTM is necessarily multi-valued. Using $Z=\mathbb{N}^{\mathbb{N}}$ allows, for example, to write the Halting problem (in form of its characteristic sequence). One guesses, for each Turing machine-input pair, an upper bound on the runtime for a halting computation. If each number is correct, this allows to

\footnotetext{
${ }^{1}$ See also [15].
} 
compute the Halting problem by simply simulating the computation for the given number of steps. On the other hand, if the input is incorrect, there will be a computation that halts after its allotted time has elapsed. This can be detected by continuing all simulations for ever.

Non-deterministic Type-2 machines are not proposed as a realistic model of computation, but-just as in the classical case - as a useful conceptual tool helping to understand deterministic computations. A novel aspect is the availability of non-trivial theorems removing non-deter-minism. Often, giving a nondeter-ministic algorithm (with compact advice space) for a function is much easier than to directly come up with a deterministic algorithm. A metatheorem ([4], [2]) then allows to remove the non-deter-minism and to obtain a deterministic algorithm. Implicitly, such an approach is exhibited in [24] by Rettinger for computability of Jordan curves, and by Galatolo, Hoyrup and Robas in [8] showing computability results for invariant measures. The present paper constitutes another application of this type.

\section{The representations}

In order to imbue a computable version of Theorem 1 with meaning, we need to clarify how the various objects are represented for the purpose of computations. The basic framework is computable analysis, laid out by Weihrauch in [32]. In particular, we will mostly work on computable metric spaces. In order to obtain a computable metric space from a separable metric space, one needs to fix a dense sequence, such that the distances are computable from the indices, this induces a computability structure on it. Every separable metric space is isomorphic to one admitting such a dense sequence, so in working on computable metric spaces we do not suffer any additional loss in generality as compared to working on separable metric spaces.

Representations of measurable sets and functions have been investigated by Brattka [1]. The representations given below are straight-forward adoptions of those used by Brattka. As foundation for the representations, we obtain from represented spaces $\mathbf{X}, \mathbf{Y}$ the space $\mathscr{C}(\mathbf{X}, \mathbf{Y})$ of continuous functions from $\mathbf{X}$ to $\mathbf{Y}$, the product space $\mathbf{X} \times \mathbf{Y}$ and the spaces of closed $\mathscr{A}(\mathbf{X})$ and open $\mathscr{O}(\mathbf{X})$ subsets of $\mathbf{X}$. Regarding these constructions, see also [22] by Pauly (based on [23, Chapter 3.2]).

Definition 2. Given a computable metric space $\mathbf{X}$, we define the space $\Delta_{2}^{0}(\mathbf{X})$ of $\Delta_{2}^{0}$ sets by identifying $\left(A_{i}, U_{i}\right)_{i \in \mathbb{N}} \in \mathscr{C}(\mathbb{N}, \mathscr{A}(\mathbf{X}) \times \mathscr{O}(\mathbf{X}))$ with the set $D:=\bigcup_{i \in \mathbb{N}} A_{i}=\bigcap_{i \in \mathbb{N}} U_{i}$. If the two sets are unequal, the corresponding $\left(A_{i}, U_{i}\right)_{i \in \mathbb{N}}$ does not represent an element of $\Delta_{2}^{0}(\mathbf{X})$.

Definition 3. Given a computable metric space $\mathbf{X}$ and a represented space $\mathbf{Y}$, we define the space $\Delta_{2}^{0}(\mathbf{X}, \mathbf{Y})$ of $\Delta_{2}^{0}$-measurable functions as the subspace of $\mathscr{C}\left(\mathscr{O}(\mathbf{Y}), \Delta_{2}^{0}(\mathbf{X})\right)$ containing functions of the form $f^{-1}$ for some $f: \mathbf{X} \rightarrow \mathbf{Y}$.

This definition of the space $\Delta_{2}^{0}(\mathbf{X}, \mathbf{Y})$ adds a uniformity constraint: Not only do we require the preimage of any open set to be a $\Delta_{2}^{0}$-set, but we require the function mapping open sets to their preimages to be continuous itself. In the case of $\Sigma_{n}^{0}$-measurable functions discussed by Brattka in [1], this constraint actually comes for free: Whenever a function $g: \mathscr{O}(\mathbf{Y}) \rightarrow \Sigma_{n}^{0}(\mathbf{X})$ is of the form $g=f^{-1}$ for some function $f: \mathbf{X} \rightarrow \mathbf{Y}$, then $g$ is already continuous. For $\Delta_{2}^{0}$-measurable functions, the situation is more complicated, though (see Section 7).

Definition 4. Given represented spaces $\mathbf{X}, \mathbf{Y}$ we define the space $\mathscr{C}^{\mathscr{A}-p w}(\mathbf{X}, \mathbf{Y})$ of $\mathscr{A}$-piecewise continuous functions by representing a function $f: \mathbf{X} \rightarrow \mathbf{Y}$ with a sequence ${ }^{2}\left(A_{i}, f_{i}\right)_{i \in \mathbb{N}} \in \mathscr{C}(\mathbb{N}, \mathscr{A}(\mathbf{X}) \times$ $\left.\mathscr{C}\left(\mathbf{A}_{i}, \mathbf{Y}\right)\right)$ such that $X=\bigcup_{i \in \mathbb{N}} A_{i}$ and $f_{i}=f_{\mid A_{i}}$.

Unlike the situation for $\Delta_{2}^{0}(\mathbf{X}, \mathbf{Y})$, one can easily verify that for admissible $\mathbf{Y}$ the space $\mathscr{C}^{\mathscr{A}-p w}(\mathbf{X}, \mathbf{Y})$ contains extensionally exactly the functions classically consider piecewise continuous.

\footnotetext{
${ }^{2}$ This occurrence of a dependent type can easily seen to be unproblematic.
} 


\section{Weihrauch reducibility and closed choice}

A convenient framework to discuss hypercomputability for general spaces is found in Weihrauch reducibility. Based on a related reducibility notion introduced by Weihrauch [30, 31], it was primarily used by Brattka, Gherardi, Marcone and Pauly [9, 20, 3, 5] to pursue computable reverse mathematics. The resulting degree structure was investigated by Brattka, Gherardi, Higuchi and Pauly [21, 4, 12]. Here we only reference the product operation $\times$, without making use of any specific properties.

Definition 5. For $f: \subseteq \mathbf{X} \rightrightarrows \mathbf{Y}, g: \subseteq \mathbf{U} \rightrightarrows \mathbf{Y}$, we say that $f$ is Weihrauch reducible to $g(f \leq \mathrm{w} g)$, iff there are computable $H, K: \subseteq\{0,1\}^{\mathbb{N}} \rightarrow\{0,1\}^{\mathbb{N}}$, such that whenever $G: \subseteq\{0,1\}^{\mathbb{N}} \rightarrow\{0,1\}^{\mathbb{N}}$ is a realizer of $g$, we find $x \mapsto H(\langle x, G K(x)\rangle)$ to be a realizer of $f$.

We say that $f$ is strongly Weihrauch reducible to $g\left(f \leq_{s W} g\right)$, if there are computable $H, K: \subseteq$ $\{0,1\}^{\mathbb{N}} \rightarrow\{0,1\}^{\mathbb{N}}$ such that whenever $G$ is a realizer of $g$, we find $H G K$ to be a realizer of $f$.

Various important Weihrauch degrees are those of closed choice principles. For a represented space $\mathbf{X}$, we consider $C_{\mathbf{X}}: \subseteq \mathscr{A}(\mathbf{X}) \rightrightarrows \mathbf{X}$ defined via $\operatorname{dom}\left(C_{\mathbf{X}}\right)=\mathscr{A}(\mathbf{X}) \backslash\{\emptyset\}$ and $x \in C_{\mathbf{X}}(A)$ iff $x \in A$. Closed choice principles are closely linked to non-deter-ministic computation:

Theorem 6 (Brattka, de Brecht \& Pauly [2, Theorem 7.2]). $f: \subseteq \mathbf{X} \rightrightarrows \mathbf{Y}$ is non-deter-ministically computable with advice space $\mathbf{Z}$ if and only if $f \leq{ }_{\mathrm{w}} C_{\mathbf{Z}}$.

The class of multivalued functions reducible to $C_{\mathbb{N}}$ is not only also classified as those non-deter-ministically computable with advice space $\mathbb{N}$, but also as those computable by a finitely revising machine (introduced by Ziegler [34, 35]) or by a generalized Turing machine allowed to make equality tests on $\{0,1\}^{\mathbb{N}}$ (introduced by Tavana and Weihrauch [28]) as can be seen following [19] by Pauly. In the present paper, we demonstrate that this class can be seen as a generalization of piecewise continuity to multivalued functions between represented spaces.

\section{The main result}

Our computable version of the Jayne-Rogers Theorem is based on the fact that evaluation for $\Delta_{2}^{0}-$ measurable functions between computable metric spaces is non-deter-ministically computable with advice space $\mathbb{N}$ (or alternatively with a finitely revising machine).

Theorem 7. Let $\mathbf{X}, \mathbf{Y}$ be computable metric spaces. The function Eval $: \Delta_{2}^{0}(\mathbf{X}, \mathbf{Y}) \times \mathbf{X} \rightarrow \mathbf{Y}$ satisfies $\operatorname{EvAL} \leq{ }_{\mathrm{W}} C_{\mathbb{N}}$.

Proof. We show that Eval is non-deter-ministically computable with advice space $\mathbb{N} \times\{0,1\}^{\mathbb{N}}$. By [2, Theorem 7.2], this implies EvAL $\leq{ }_{\mathrm{W}} C_{\mathbb{N} \times\{0,1\}^{\mathrm{N}}}$. By [2, Corollary 4.9] we have $C_{\mathbb{N} \times\{0,1\}^{\mathbb{N}}} \leq \mathrm{W} C_{\mathbb{N}} \times$ $C_{\{0,1\}^{\mathbb{N}}}$. As EvAL is single-valued, we can then invoke [2, Theorem 5.1] to conclude EvAL $\leq{ }_{\mathrm{W}} C_{\mathbb{N}}$.

We regard $\mathbf{Y}$ as a subspace of the Hilbert cube $\mathbf{H}:=\widehat{0,1}]$, and assume the latter to be represented by the total representation $\delta_{\mathbf{H}}:\{0,1\}^{\mathbb{N}} \rightarrow \widehat{[0,1]}([2$, Proposition 4.1]), and the former by a suitable restriction $\delta_{\mathbf{Y}}$ of $\delta_{\mathbf{H}}$. The map restrict : $\mathscr{O}(\mathbf{H}) \rightarrow \mathscr{O}(\mathbf{Y})$ defined via restrict $(U)=U \cap Y$ is trivially computable.

A non-deter-ministic algorithm to compute Eval on input $f \in \Delta_{2}^{0}(\mathbf{X}, \mathbf{Y})$ and $x \in \mathbf{X}$ guesses $y \in$ $\{0,1\}^{\mathbb{N}}$ and $n \in \mathbb{N}$. The output in case of a successful guess is $y$.

As $\mathbf{H}$ is computably $T_{2}$, one can compute $\left\{\delta_{\mathbf{H}}(y)\right\} \in \mathscr{A}(\mathbf{H})$ from $y$, which has the same names as $H \backslash\left\{\delta_{\mathbf{H}}(y)\right\} \in \mathscr{O}(\mathbf{H})$. Hence, we can compute $Y \backslash\left\{\delta_{\mathbf{Y}}(y)\right\}=\operatorname{restrict}\left(H \backslash\left\{\delta_{\mathbf{H}}(y)\right\}\right) \in \mathscr{O}(\mathbf{Y})$, where we understand $\left\{\delta_{\mathbf{Y}}(y)\right\}=\emptyset$ for $y \notin \operatorname{dom}\left(\delta_{\mathbf{Y}}\right)$. 
The information available on $f$ then allows to compute $f^{-1}\left(Y \backslash\left\{\delta_{\mathbf{Y}}(y)\right\}\right) \in \Delta_{2}^{0}(\mathbf{X})$, in particular, we can access $f^{-1}\left(Y \backslash\left\{\delta_{\mathbf{Y}}(y)\right\}\right) \in \Pi_{2}^{0}(\mathbf{X})$, and then also $f^{-1}\left(\left\{\delta_{\mathbf{Y}}(y)\right\}\right) \in \Sigma_{2}^{0}(\mathbf{X})$.

With $\mathbf{X}$ being a metric space, a $\sum_{2}^{0}$-set is the union of countably many closed sets, so we find $f^{-1}\left(\left\{\delta_{\mathbf{Y}}(y)\right\}\right)=\bigcup_{i \in \mathbb{N}} A_{i}$ with $A_{i} \in \mathscr{A}(\mathbf{X})$. We simultaneously test $x \in A_{i}$ ? for all $i \in \mathbb{N}$. If ever $x \notin A_{i}$ is confirmed for all $i \leq n$, the guess is rejected.

If a guess $(y, n)$ is never rejected, then there is some $i \leq n$ with $x \in A_{i} \subseteq\left(\bigcup_{i \in \mathbb{N}} A_{i}\right)=f^{-1}\left(\left\{\delta_{\mathbf{y}}\right\}\right)$, hence $f(x)=\delta_{\mathbf{Y}}(y)$ and the output is correct. Also, if $\delta_{\mathbf{Y}}(y)=f(x)$, then $x \in\left(\bigcup_{i \in \mathbb{N}} A_{i}\right)$, so there is some $N \in \mathbb{N}$ with $x \in A_{N}$. But then $(y, N)$ can never be rejected.

A multivalued function $f: \mathbf{X} \rightrightarrows \mathbf{Y}$ reducible to $C_{\mathbb{N}}$ is clearly computable when restricted to those inputs where any fixed $n \in \mathbb{N}$ is a valid answer to the oracle question to $C_{\mathbb{N}}$. Any such set is a closed subset of $\operatorname{dom}\left(\delta_{X}\right)$, however, we will need the following lemma to lift these to closed subsets of $\mathbf{X}$.

Given a set $A \subseteq\{0,1\}^{\mathbb{N}}$ and a represented space $\mathbf{X}$, we can obtain the represented space $\mathbf{X}_{A}$ by restricting the representation $\delta_{X}$ to $A$. This can be seen as a generalization of the subspace construction: If $A=\delta_{X}^{-1} \delta_{X}[A]$, then $\mathbf{X}_{A}=\mathbf{X}_{\mid \delta_{X}[A]}$ actually is a subspace of $\mathbf{X}$.

Lemma 8. Let $\mathbf{X}, \mathbf{Y}$ be computable metric spaces, and let $\delta_{\mathbf{X}}$ be proper.

1. The map $\delta_{X}[]: \mathscr{A}\left(\{0,1\}^{\mathbb{N}}\right) \rightarrow \mathscr{A}(\mathbf{X})$ is well-defined and computable $]^{3}$

2. Given $A \in \mathscr{A}\left(\{0,1\}^{\mathbb{N}}\right)$ and $f \in \mathscr{C}\left(\mathbf{X}_{A}, \mathbf{Y}\right)$ one can compute $f \in \mathscr{C}\left(\mathbf{X}_{\mid \delta_{X}[A]}, \mathbf{Y}\right)$.

Proof. 1. As every computable metric space $\mathbf{X}$ is computably $T_{2}$, we can compute the compact singleton $\{x\}$ from $x \in \mathbf{X}$. As $\delta_{X}$ is assumed proper, $\delta_{X}^{-1}(\{x\})$ is compact, and moreover, can be computed from $x$ as a compact set. The intersection of a closed set and a compact set is uniformly compact, so from $x$ we compute $\delta_{X}^{-1}(\{x\}) \cap A$ as a compact set. Emptyness for compact sets is semidecidable, and $x \mapsto \operatorname{IsEmpty}\left(\delta_{X}^{-1}(\{x\}) \cap A\right)$ realizes $\delta_{\mathbf{X}}[A]$.

2. As before, we can compute $\delta_{X}^{-1}(\{x\}) \cap A$ from $x \in \mathbf{X}$, which just is the compact singleton $\{x\} \in$ $\mathscr{K}\left(\mathbf{X}_{A}\right)$. Images of compact sets under continuous functions are uniformly compact, so we obtain $\{f(x)\} \in \mathscr{K}(\mathbf{Y})$. Computable metric spaces are admissible, so from a compact singleton the value can be obtain, which gives us $f(x) \in \mathbf{Y}$. This treatment is uniform in $f$, thus yields the claim.

Theorem 9. Let $\mathbf{X}, \mathbf{Z}$ be computable metric spaces, and $\mathbf{Y}$ a represented space. If $f: \mathbf{X} \times \mathbf{Y} \rightarrow \mathbf{Z}$ satisfies $f \leq_{\mathrm{W}} C_{\mathbb{N}}$, then $y \mapsto(x \mapsto f(x, y)): \mathbf{Y} \rightarrow \mathscr{C}^{\mathscr{A}-p w}(\mathbf{X}, \mathbf{Z})$ is computable.

Proof. Let the reduction $f \leq \mathrm{w} C_{\mathbb{N}}$ be witnessed by $H, K$, and let $\mathscr{A}(\mathbb{N})$ be represented by $\psi^{\mathbb{N}}$. From $n \in \mathbb{N}$ and $q \in \operatorname{dom}\left(\delta_{Y}\right)$ one can compute $p \mapsto\left(n \in \psi^{\mathbb{N}} K(\langle p, q\rangle)\right)$, which realizes a closed set $A_{n, q} \in$ $\mathscr{A}\left(\{0,1\}^{\mathbb{N}}\right)$. The map $p \mapsto \delta_{Z} H\left(\langle\langle p, q\rangle, n)\right.$ now realizes $f_{A_{n, q}} \in \mathscr{C}\left(\mathbf{X}_{A_{n, q}}, \mathbf{Z}\right)$. Using Lemma 8 , both the closed cover and the partial realizers can be lifted from $\{0,1\}^{\mathbb{N}}$ to $\mathbf{X}$.

Theorem 10. For computable metric spaces $\mathbf{X}, \mathbf{Y}$ the maps id $: \Delta_{2}^{0}(\mathbf{X}, \mathbf{Y}) \rightarrow \mathscr{C}^{\mathscr{A}-p w}(\mathbf{X}, \mathbf{Y})$ and id : $\mathscr{C}^{\mathscr{A}-p w}(\mathbf{X}, \mathbf{Y}) \rightarrow \Delta_{2}^{0}(\mathbf{X}, \mathbf{Y})$ are well-defined and computable.

Proof. The first part of the claim follows from combining Theorems 7, 9, For the second part, we need to show that from an open set $U \subseteq \mathbf{Y}$ and a piecewise continuous function $f: \mathbf{X} \rightarrow \mathbf{Y}$ we can compute the $\Delta_{2}^{0}$-set $f^{-1}(U)$.

\footnotetext{
${ }^{3}$ Note that this does not follow directly from the fact that continuous images of compact sets are compact, together with compactness of $\{0,1\}^{\mathbb{N}}$ and the $T_{2}$-property of $\mathbf{X}$ (cf. [22]), as $\delta_{\mathbf{X}}$ may very well be partial.
} 
Let $\left(A_{i}\right)_{i \in \mathbb{N}}$ be the closed cover of $\mathbf{X}$ coming with $f$. As any $f_{\mid A_{i}}$ is given as a continuous function, we can compute all $f_{\mid A_{i}}^{-1}(U) \in \mathscr{O}\left(\mathbf{A}_{i}\right)$. Invoking Lemma 8 (1), we can extend the open set $f_{\mid A_{i}}^{-1}(U)$ to an open set $U_{i} \in \mathscr{O}(\mathbf{X})$, such that $f_{\mid A_{i}}^{-1}(U)=A_{i} \cap U_{i}$. In total, we find $f^{-1}(U)=\bigcup_{i \in \mathbb{N}}\left(A_{i} \cap U_{i}\right)$. In a computable metric space, any open set $U_{i}$ can be represented as a union $\bigcup_{j \in \mathbb{N}} \bar{U}_{i}^{j}$, hence we obtain $f^{-1}(U)=\bigcup_{i, j \in \mathbb{N}} A_{i} \cap \bar{U}_{i}^{j}$,

To express $f^{-1}(U)$ as a countable intersection of open sets, consider $f^{-1}\left(U^{C}\right)$. Any $f_{\mid A_{i}}^{-1}\left(U^{C}\right)=: B_{i}$ is closed in $A_{i}$, hence in $\mathbf{X}$. We have that $f^{-1}\left(U^{C}\right)=\bigcup_{i \in \mathbb{N}} B_{i}$ implies $f^{-1}(U)=\bigcap_{i \in \mathbb{N}} B_{i}^{C}=\bigcap_{i \in \mathbb{N}}\left(A_{i}^{C} \cup\right.$ $\left.f_{\mid A_{i}}^{-1}(U)\right)$, which is computable from the givens.

Corollary 11. For computable metric spaces $\mathbf{X}$, $\mathbf{Y}$ we find that the map EvAL : $\mathscr{C}^{\mathscr{A}-p^{w}}(\mathbf{X}, \mathbf{Y}) \times \mathbf{X} \rightarrow \mathbf{Y}$ satisfies EvAL $\leq \mathrm{w} C_{\mathbb{N}}$.

We can call a function $f: \mathbf{X} \rightarrow \mathbf{Y}$ effectively $\Delta_{2}^{0}$-measurable, if it has a computable name in $\Delta_{2}^{0}(\mathbf{X}, \mathbf{Y})$. This means that one can compute $\Delta_{2}^{0}$-preimages of open sets without additional information. Likewise, a function with a computable name in $\mathscr{C}^{\mathscr{A}-p w}(\mathbf{X}, \mathbf{Y})$ is called piecewise computable, this entails the existence of a countable cover by computably closed sets such that the corresponding restrictions are computable. As computable functions map computable elements to computable elements, we obtain the next corollary:

Corollary 12. A function $f: \mathbf{X} \rightarrow \mathbf{Y}$ between computable metric spaces is effectively $\Delta_{2}^{0}$-measurable, if and only if it is piecewise computable, if and only if $f \leq_{\mathrm{W}} C_{\mathbb{N}}$.

\section{Markov-effective $\Delta_{2}^{0}$-measurability}

Besides the notion of effective $\Delta_{2}^{0}$-measurability used for Corollary 12 , there is a second possible definition. Given computable metric spaces, we can fix effective partial enumerations $\left(U_{n}\right)_{n \in \mathbb{N}}$ of the computably open subsets of $\mathbf{Y}$ and $\left(D_{n}\right)_{n \in \mathbb{N}}$ of the computably $\Delta_{2}^{0}$ sets in $\mathbf{X}$. Then we call $f: \mathbf{X} \rightarrow \mathbf{Y}$ Markoveffectively $\Delta_{2}^{0}$-measurable, if there is a partial computable function $P: \subseteq \mathbb{N} \rightarrow \mathbb{N}$, such that whenever $U_{n}$ is an open subset of $\mathbf{X}$, we find $D_{P(n)}$ to be the $\Delta_{2}^{0}$ set $f^{-1}\left(U_{n}\right)$. This is the notion of effectivity that might be chosen in recursive analysis or effective descriptive set theory to capture $\Delta_{2}^{0}$-measurability.

We shall demonstrate that Markov-effective $\Delta_{2}^{0}$-measurability can be characterized via Weihrauch reducibility in a similar fashion to Corollary 12, however, unlike its more uniform counterpart it is not related to piecewise computability (or finitely revising computability), but rather to low computability.

Recall from recursion theory that $p \in\{0,1\}^{\mathbb{N}}$ is called low, iff the Turing jump of $p$ is the Halting problem, i.e., as simple as possible. In [2], Brattka, de Brecht and Pauly suggested a uniform counterpart: We call $f: \mathbf{X} \rightrightarrows \mathbf{Y}$ low computable, if from any name $p$ for $x \in \operatorname{dom}(f)$, we can compute a sequence converging to the Turing jump of some name $q$ for some $y \in f(x)$.

We restrict the considerations to Cantor space for now. Here, the effective enumeration $\left(U_{n}\right)_{n \in \mathbb{N}}$ of the computably open sets can be chosen total, and the Turing jump $J:\{0,1\}^{\mathbb{N}} \rightarrow\{0,1\}^{\mathbb{N}}$ defined via $J(p)(i)=1$ iff $p \in U_{i}$.

Theorem 13. $f: \subseteq\{0,1\}^{\mathbb{N}} \rightarrow\{0,1\}^{\mathbb{N}}$ is Markov-effectively $\Delta_{2}^{0}$-measurable if and only if it is low computable.

Proof. $\Rightarrow$ Given a function $f$, if we have a uniform way of computing from an index $n$ a $\Delta_{2}^{0}$-name for $f^{-1}\left(U_{n}\right)$, then we can create a limit computable function that, on input $p$, converges to 1 if 
$f(p) \in U_{n}$ and converges to 0 if $f(p) \notin U_{n}$. So we can limit compute the $n$-th place of the Jump of $f(p)$. If we can compute this uniformly in $n$, then by doing it all in parallel we can low-compute $f$.

$\Leftarrow$ On the other hand, if we can low-compute $f$, then we have a limit computable function that converges to 1 if $f(p) \in U_{n}$ and 0 if $f(p) \notin U_{n}$ (by just looking at the $n$-th place of our low-computation of $f$ ). This implies that the preimage of $U_{n}$ under $f$ is effectively $\Sigma_{2}^{0}$ (i.e., the effective union of countably many computably closed sets) and the preimage of the complement of $U_{n}$ under $f$ is also effectively $\Sigma_{2}^{0}$, hence the preimage of $U_{n}$ is effectively $\Delta_{2}^{0}$.

Low computability was characterized in [2, Theorem 8.10] in terms of the function $\mathfrak{L}: \subseteq \mathscr{C}\left(\mathbb{N}, \mathbb{N}^{\mathbb{N}}\right) \rightarrow$ $\mathbb{N}^{\mathbb{N}}$ defined via $\mathfrak{L}\left(\left(p_{i}\right)_{i \in \mathbb{N}}\right)=q$ iff $\lim _{i \rightarrow \infty} p_{i}=J(q)$. One finds that any $f$ is low computable if and only if $f \leq_{s W} \mathfrak{L}$ holds.

Corollary 14. $f: \subseteq\{0,1\}^{\mathbb{N}} \rightarrow\{0,1\}^{\mathbb{N}}$ is Markov-effectively $\Delta_{2}^{0}$-measurable if and only if $f \leq_{s W} \mathfrak{L}$.

Fact 15. $\mathfrak{L} \not \mathbb{W}_{\mathrm{W}} C_{\mathbb{N}}$.

Corollary 16. Markov-effective $\Delta_{2}^{0}$-measurability does not imply effective $\Delta_{2}^{0}$-measurability.

Corollary 17. There is a Markov-effectively $\Delta_{2}^{0}$-measurable function that is not even piecewise continuous.

We point out that Higuchi and Kihara [11] have independently obtained a similar result to Theorem 13 which holds for Markov-effective $\Delta_{n}^{0}$-measurability and $\operatorname{low}_{n}$-computability (although their terminology differs from ours).

\section{Computable vs. classical Jayne-Rogers theorem}

As remarked after Definition 3 , it is not guaranteed that the represented space $\Delta_{2}^{0}(\mathbf{X}, \mathbf{Y})$ actually contains all $\Delta_{2}^{0}$-measurable functions. In principle, it is conceivable that some $f^{-1}: \mathscr{O}(\mathbf{Y}) \rightarrow \Delta_{2}^{0}(\mathbf{X})$ is welldefined, i.e., the inverse of a function $f: \mathbf{X} \rightarrow \mathbf{Y}$, yet lacks continuous realizers. As a consequence, the classical Jayne-Rogers Theorem does not follow directly from its computable counterpart.

However, for spaces $\mathbf{X}, \mathbf{Y}$ in its scope, the classical Jayne-Rogers Theorem states that all $\Delta_{2}^{0}$-measurable functions are elements in the space $\mathscr{C}^{\mathscr{A}-p w}(\mathbf{X}, \mathbf{Y})$ - hence, by the computable Jayne-Rogers Theorem (Theorem 10), they are elements of $\Delta_{2}^{0}(\mathbf{X}, \mathbf{Y})$.

The classical Jayne-Rogers Theorem is not necessary for $\Delta_{2}^{0}(\mathbf{X}, \mathbf{Y})$ to contain all $\Delta_{2}^{0}$-measurable functions, though. Consider the space $\omega+1=\{A \in \mathscr{O}(\mathbb{N}) \mid n \in A \wedge m \geq n \Rightarrow m \in A\}$ with the Scott topology. Now $\mathscr{O}(\omega+1)$ is countable, and there is a computable injection $\imath: \mathscr{O}(\omega+1) \rightarrow \mathbb{N}^{\nabla}\left(\mathbb{N}^{\nabla}\right.$ are the natural number with the finitely revising representation). Using a list of all preimages of open sets, we find for any $\Delta_{2}^{0}$-measurable function $f: \mathbb{N}^{\mathbb{N}} \rightarrow \omega+1$ that $f^{-1}: \mathscr{O}(\omega+1) \rightarrow\left(\Delta_{2}^{0}\left(\mathbb{N}^{\mathbb{N}}\right)\right)^{\nabla}$ is continuous. As $\left(\Delta_{2}^{0}\left(\mathbb{N}^{\mathbb{N}}\right)\right)^{\nabla}$ and $\Delta_{2}^{0}\left(\mathbb{N}^{\mathbb{N}}\right)$ are isomorphic, $\Delta_{2}^{0}\left(\mathbb{N}^{\mathbb{N}}, \omega+1\right)$ encompasses all $\Delta_{2}^{0}$-measurable functions from $\mathbb{N}^{\mathbb{N}}$ to $\omega+1$.

Consider the function $e: \mathbb{N}^{\mathbb{N}} \rightarrow(\omega+1)$ defined via $e(p)=\{i \geq \max \{p(j) \mid j \in \mathbb{N}\}+1\}$ if $\max \{p(j) \mid$ $j \in \mathbb{N}\}$ exists and is even, $e(p)=\{i \geq \max \{p(j) \mid j \in \mathbb{N}\}-1\}$ if $\max \{p(j) \mid j \in \mathbb{N}\}$ exists and is odd, and $e(p)=\mathbb{N}$ if $p$ is unbounded. Then $e$ is $\Delta_{2}^{0}$-measurable, yet not piecewise continuous. Thus, the Jayne-Rogers Theorem cannot be extended to $\omega+1$ as codomain.

As just demonstrated, it is in principle possible to prove that the space $\Delta_{2}^{0}(\mathbf{X}, \mathbf{Y})$ does contain all $\Delta_{2}^{0}-$ measurable functions for certain spaces $\mathbf{X}, \mathbf{Y}$ without resorting to the classical Jayne-Rogers Theorem. 
This gives hope that a more general result of this form, together with the computable Jayne-Rogers Theorem could be used as a simple proof for a (generalization of) the classical Jayne-Rogers Theorem.

It is worthwhile pointing out the analogy to the Kreitz-Weihrauch representation theorem for admissible representations proving that the space $\mathscr{C}(\mathbf{X}, \mathbf{Y})$ contains all the (topologically) continuous functions (see also [25] by Schröder, [22] by Pauly). In the context of $\Sigma_{n}^{0}$-measurable functions, this connection has been explored in detail by de Brecht and Yamamoto in [7].

\section{Generalizing the main result}

The proof of Theorem 7, the center piece of our main result, makes no use of properties exclusive to metric spaces, and hence can be extended to more general spaces. The precise characterization of the suitable spaces is left for future work, however, we do have some limits how far the Jayne-Rogers Theorem can be extended.

In [13], Jayne and Rogers provide a counterexample with a metric, but non absolute Souslin- $\mathfrak{F}$ domain, and a discrete uncountable metric space as codomain assuming Martin's axiom. The latter is not available in a computable context, in particular, the absolute Souslin- $\mathfrak{F}$ condition is irrelevant for us.

A candidate condition is the $T_{D}$ separation axiom. A topological space is $T_{D}$, if any singleton is the intersection of an open and a closed set. A prototypic space failing the $T_{D}$ criterion is $\omega+1$, hence the non-piecewise continuous function $e \in \Delta_{2}^{0}\left(\mathbb{N}^{\mathbb{N}}, \omega+1\right)$ introduced in the previous section bars an extension of the Jayne-Rogers Theorem to non- $T_{D}$ spaces.

On the other hand, a computable $T_{D}$ property suffices instead of the computable $T_{2}$ property employed in the proof of Theorem 7. A naive definition of computably $T_{D}$ requiring that from any singleton $x \in \mathbf{X}$ one can compute a pair $\left(A_{x}, U_{x}\right) \in \mathscr{A}(\mathbf{X}) \times \mathscr{O}(\mathbf{X})$ with $A_{x} \cap U_{x}=\{x\}$ turns out to be equivalent to computably $T_{2}$. However, allowing computation with finitely many mindchanges here, or, alternatively, requiring the computability of $x \mapsto\{x\}: \mathbf{X} \rightarrow \Delta_{2}^{0}(\mathbf{X})$ suffices for Theorem 7 . The question which spaces embed into a computable $T_{D}$-space with a total Cantor-representation remains unresolved, though.

Another potential direction of generalization requires a better understanding of the interaction of two computational models, namely non-deterministic and limit machines. This could lead to a classification of the Weihrauch degree of function evaluation for functions where the preimages of open sets are $\Delta_{n}^{0}$ also for $n>2$.

In a non-uniform way Higuchi and Kihara [11] made some progress in understanding the higher levels of effective measurability. They prove that a function where the preimages of $\Sigma_{n}^{0}$-sets effectively are $\Sigma_{n}^{0}$-sets will necessarily be non-uniformly computable.

In general, the results presented here could be an indication that computable descriptive set theory could be developed relying heavily on Type- 2 models of computation. That descriptive set theory has an underlying algorithmic structure is already evident from the rôle of games in this field, exhibited, e.g., in [29] by Wadge and [26] by Semmes, which is generalized significantly in [17] by Motto-Ros. Computational models have some advantages over games, such as straight-forward closure properties under composition, that might provide additional usefulness to such an approach.

\section{References}

[1] Vasco Brattka (2005): Effective Borel measurability and reducibility of functions. Mathematical Logic Quarterly 51(1), pp. 19-44, doi $10.1002 /$ malq.200310125. 
[2] Vasco Brattka, Matthew de Brecht \& Arno Pauly (2012): Closed Choice and a Uniform Low Basis Theorem. Annals of Pure and Applied Logic 163(8), pp. 968-1008, doi:10.1016/j.apal.2011.12.020

[3] Vasco Brattka \& Guido Gherardi (2011): Effective Choice and Boundedness Principles in Computable Analysis. Bulletin of Symbolic Logic 1, pp. 73 - 117, doi $10.2178 / \mathrm{bsl} / 1294186663$.

[4] Vasco Brattka \& Guido Gherardi (2011): Weihrauch Degrees, Omniscience Principles and Weak Computability. Journal of Symbolic Logic 76, pp. 143 - 176, doi:10.2178/jsl/1294170993.

[5] Vasco Brattka, Guido Gherardi \& Alberto Marcone (2012): The Bolzano-Weierstrass Theorem is the Jump of Weak König's Lemma. Annals of Pure and Applied Logic 163(6), pp. 623-625, doi $10.1016 /$ j.apal.2011.10.006.

[6] Vasco Brattka \& Arno Pauly (2010): Computation with Advice. In Xizhong Zheng \& Ning Zhong, editors: Proceedings Seventh International Conference on Computability and Complexity in Analysis, Electronic Proceedings in Theoretical Computer Science 24, pp. 41-55, doi:10.4204/EPTCS.24.9.

[7] Matthew de Brecht \& Akihiro Yamamoto (2009): $\Sigma_{\alpha}^{0}$-Admissible Representations (Extended Abstract). In Andrej Bauer, Peter Hertling \& Ker-I Ko, editors: Sixth International Conference on Computability and Complexity in Analysis, CCA 2009, August 18-22, 2009, Ljubljana, Slovenia, Open Access Series in Informatics 11, Schloss Dagstuhl, pp. 119-130, doi:10.4230/OASIcs.CCA.2009.2264

[8] Stefano Galatolo, Mathieu Hoyrup \& Christobal Robas (2011): Dynamics and abstract computability: computing invariant measures. Discrete and Continuous Dynamical Systems 29(1), pp. 193-212, doi $10.3934 /$ dcds.2011.29.193.

[9] Guido Gherardi \& Alberto Marcone (2009): How incomputable is the separable Hahn-Banach theorem? Notre Dame Journal of Formal Logic 50(4), pp. 393-425, doi:10.1215/00294527-2009-018

[10] Joel David Hamkins \& Andy Lewis (2000): Infinite Time Turing Machines. Journal of Symbolic Logic 65(2), pp. 567-604, doi: $10.2307 / 2586556$.

[11] Kojiro Higuchi \& Takayuki Kihara: Inside the Muchnik Degrees: Discontinuity, Learnability, and Constructivism. preprint.

[12] Kojiro Higuchi \& Arno Pauly (2013): The degree-structure of Weihrauch-reducibility. Logical Methods in Computer Science 9(2), doi 10.2168/LMCS-9(2:2)2013.

[13] J. E. Jayne \& C. A. Rogers (1984): Piece-wise closed functions. Mathematische Annalen 255, pp. 499-518, doi $10.1007 / \mathrm{BF} 01451930$.

[14] J.E. Jayne \& C.A. Rogers (1982): First level Borel functions and isomorphisms. Journal de Mathématiques Pures et Appliquées 61, pp. 177-205.

[15] Miroslav Kačena, Luca Motto Ros \& Brian Semmes (2012): Some observations on 'A New Proof of a Theorem of Jayne and Rogers'. Real Analysis Exchange 38(1), pp. 121-132.

[16] Alexander S. Kechris (1995): Classical Descriptive Set Theory. Graduate Texts in Mathematics 156, Springer, doi $10.1007 / 978-1-4612-4190-4$.

[17] Luca Motto Ros (2011): Game representations of classes of piecewise definable functions. Mathematical Logic Quarterly 57(1), pp. 95-112, doi:10.1002/malq.200910124

[18] Luca Motto Ros \& Brian Semmes (2009): A New Proof of a Theorem of Jayne and Rogers. Real Analysis Exchange 35(1), pp. 195-204.

[19] Arno Pauly (2009): Infinite Oracle Queries in Type-2 Machines (Extended Abstract). arXiv:0907.3230v1.

[20] Arno Pauly (2010): How Incomputable is Finding Nash Equilibria? Journal of Universal Computer Science 16(18), pp. 2686-2710, doi:10.3217/jucs-016-18-2686.

[21] Arno Pauly (2010): On the (semi)lattices induced by continuous reducibilities. Mathematical Logic Quarterly 56(5), pp. 488-502, doi $10.1002 /$ malq.200910104.

[22] Arno Pauly (2012): Compactness and Separation for Represented Spaces. http://arxiv.org/abs/1204.3763. 
[23] Arno Pauly (2012): Computable Metamathematics and its Application to Game Theory. Ph.D. thesis, University of Cambridge.

[24] Robert Rettinger (2011): Compactness and the Effectivity of Uniformization. Talk at CCA 2011.

[25] Matthias Schröder (2002): Extended admissibility. Theoretical Computer Science 284(2), pp. 519-538, doi $10.1016 /$ S0304-3975(01)00109-8.

[26] Brian Semmes (2009): A Game for the Borel Functions. Ph.D. thesis, University of Amsterdam.

[27] S. Solecki (1998): Decomposing Borel sets and functions and the structure of Baire class 1 functions. Journal of the American Mathematical Society 11(3), pp. 521-550, doi:10.1090/S0894-0347-98-00269-0.

[28] Nazanin Tavana \& Klaus Weihrauch (2011): Turing machines on represented sets, a model of computation for analysis. Logical Methods in Computer Science 7, pp. 1-21, doi:10.2168/LMCS-7(2:19)2011.

[29] William W. Wadge (1972): Degrees of complexity of subsets of the Baire space. Notices of the American Mathematical Society, pp. 714-715.

[30] Klaus Weihrauch (1992): The degrees of discontinuity of some translators between representations of the real numbers. Informatik Berichte 129, FernUniversität Hagen, Hagen.

[31] Klaus Weihrauch (1992): The TTE-interpretation of three hierarchies of omniscience principles. Informatik Berichte 130, FernUniversität Hagen, Hagen.

[32] Klaus Weihrauch (2000): Computable Analysis. An Introduction. Texts in Theoretical Computer Science, Springer-Verlag, doi:10.1007/978-3-642-56999-9.

[33] Martin Ziegler (2005): Computability and continuity on the real arithmetic hierarchy and the power of type-2 nondeterminism. In Barry S. Cooper, Benedikt Löwe \& Leen Torenvliet, editors: New Computational Paradigms, First Conference on Computability in Europe, CiE 2005, Amsterdam, The Netherlands, June 8-12, 2005, Proceedings, Lecture Notes in Computer Science 3526, Springer, pp. 562-571, doi:10.1007/11494645_68

[34] Martin Ziegler (2007): Real Hypercomputation and Continuity. Theory of Computing Systems 41, pp. 177 - 206, doi:10.1007/s00224-006-1343-6.

[35] Martin Ziegler (2007): Revising Type-2 Computation and Degrees of Discontinuity. Electronic Notes in Theoretical Computer Science 167, pp. 255-274, doi:10.1016/j.entcs.2006.08.015. 